\title{
Alginate/chitosan nanoparticles for encapsulation and controlled release of vitamin $B_{2}$
}

\author{
Maria A. Azevedo, Ana I. Bourbon, António A. Vicente, Miguel A. Cerqueira* \\ Centre of Biological Engineering, University of Minho, Campus de Gualtar, 4710-057 Braga, Portugal
}

\section{A R T I C L E I N F O}

\section{Article history:}

Received 12 February 2014

Received in revised form 8 May 2014

Accepted 14 May 2014

Available online 23 May 2014

\section{Keywords:}

Nanoencapsulation

Vitamin $B_{2}$

Biopolymers

\begin{abstract}
A B S T R A C T
This work aims at evaluating encapsulation and controlled release of vitamin $B_{2}$ from alginate/chitosan nanoparticles. Ionotropic polyelectrolyte pre-gelation was used as production method being chitosan and alginate used as main materials. Nanoparticles were characterized in terms of average size, polydispersity index (PDI), zeta potential and vitamin entrapment efficiency. The average size for alginate/chitosan nanoparticles was $119.5 \pm 49.9 \mathrm{~nm}$ for samples without vitamin $\mathrm{B}_{2}$ and $104.0 \pm 67.2 \mathrm{~nm}$ with the encapsulation of vitamin $B_{2}$, presenting a PDI of $0.454 \pm 0.066$ and $0.319 \pm 0.068$, respectively. The nanoparticles showed encapsulation efficiency and loading capacity values of $55.9 \pm 5.6 \%$ and $2.2 \pm 0.6 \%$, respectively. Release profiles were evaluated at different conditions showing that the polymeric relaxation was the most influent phenomenon in vitamin $B_{2}$ release. In order to study their stability nanoparticles were stored at $4{ }^{\circ} \mathrm{C}$ being particles sizes and PDI evaluated during 5 months showing the results that vitamin $\mathrm{B}_{2}$-loaded nanoparticles are more stable (in terms of size and PDI) than nanoparticles without vitamin $\mathrm{B}_{2}$.
\end{abstract}

(c) 2014 Elsevier B.V. All rights reserved.

\section{Introduction}

Food industry pays special attention to vitamins due their unique properties, e.g. essential for growth, development and normal maintenance of human organism [1,2]. In general, vitamins are defined as organic molecules of small dimension and low molecular weight, being divided into liposoluble and water-soluble vitamins according to their solubility [2,3]. Furthermore, humans do not have the capacity to synthesize vitamins, except vitamin $D$ and $B_{3}$, meaning that they must obtain vitamins through external sources $[1,2]$. Vitamin $B_{2}$ is a flavin, water-soluble compound and is present in animal and plant cells (e.g. wheat bran, milk and milk products, eggs, meats and vegetables) $[1,2,4]$.

One of the present challenges for the use of vitamins by food industry is their high sensibility and low stability to inadequate environmental conditions (e.g. temperature, oxygen, light and moisture), leading to the search for new strategies for their use. One of the presented solutions to keep stability of vitamins is nanoencapsulation [5,6]. Nanocapsules are constituted by an external cavity consisting of a polymeric membrane and an internal part composed by a liquid or polymeric matrix that contains the

\footnotetext{
* Corresponding author. Tel.: +351253601 962; fax: +351253678986

E-mail addresses: miguelcerqueira@deb.uminho.pt, miguelribeirocerqueira@gmail.com (M.A. Cerqueira).
}

active compound $[7,8]$. The selection of an appropriate method of encapsulation is an important step in order to have nanoparticles with the desired performance and functionality. The selected method will depend on the physicochemical character of the polymer, encapsulated bioactive compound and the desired properties of the nanoparticles (e.g. particle size, particle size distribution, surface area, shape, solubility, encapsulation efficiency and release mechanism) [9-11]. Besides, in order to improve the efficiency and stability of nanostructures, it is also essential to find adequate materials; in the particular case of foods, the replacement of nonfood-grade materials by bio-based and biodegradable food-grade materials is of relevance. Biopolymers, such as polysaccharides, are one of the possibilities due their distinct advantages of biodegradability, edibility and lack of toxicity [12].

Alginate is a hydrophilic polysaccharide extracted from marine brown algae of the Phaeophyta family. It is a linear biopolymer composed by two uronic acids, 1,4-linked- $\beta$-D-mannuronic acid (M) and $\alpha$-L-guluronic acid ( $\mathrm{G}$ ), being carboxylic groups from uronic acids responsible by their negative charge. This biopolymer is a non-toxic, biocompatible, biodegradable and presents mucoadhesive properties, being approved for pharmaceutical and food applications [13-15]. The solubility of alginate in water depends on the associated cations, i.e. sodium alginate is soluble in water but when a solution with multivalent cations (e.g. calcium $-\mathrm{Ca}^{2+}$ ) is used the biopolymer can form a reversible gel $[13,14,16]$. 
Chitosan is a cationic polysaccharide derived from the $\mathrm{N}$ deacetylation of chitin, the second most abundant natural biopolymer. It is a linear copolymer composed by repeating units of 2-amino-2-deoxy- $\beta$-D-glucan with glycosidic linkages, where the amine groups confer to chitosan special properties (e.g. high charge density, readily available for chemical reactions and salt formation with salts) $[13,17]$. Chitosan presents three types of reactive functional groups: an amino group as well as both primary and secondary hydroxyl groups at the C-2, C-3 and C- 6 position, respectively [18]. The position of free amino and $\mathrm{N}$-acetyl groups is responsible by the solubility of chitosan; nevertheless, chitosan solubility is improved with aqueous acids (e.g. formic acid and acetic acid) $[17,19,20]$. Chitosan is biocompatible, biodegradable, nontoxic, with significant adsorption and muchoadhesive properties and antifungal activity. Due to these properties, chitosan has a great potential for food, environmental and pharmaceutical applications $[16,19,21,22]$.

The main objective of this work was the development and characterization of a biopolymer-based nanoparticle for the encapsulation of vitamin $B_{2}$ and assessment of vitamin release from it. The main challenges were the encapsulation of vitamin $B_{2}$ into an alginate/chitosan nanoparticle and their controlled release in different conditions. Although this type of nanoparticles has been studied, in this work the alginate/chitosan nanoparticles were applied to the encapsulation of vitamin $B_{2}$ aiming their application in food industry, which may be an added value for this field.

\section{Materials and methods}

\subsection{Materials}

Sodium alginate (Manutex RSX) with $\mathrm{MW} \approx 15,900$ Da and viscosity $\approx 200 \mathrm{cp}$ ( $1 \%$ aqueous solution with Brookfield Model LV $60 \mathrm{rpm}$ at $25^{\circ} \mathrm{C}$ ) from Laminaria hyperborea was purchased from CP Kelco International, Ltd. (Portugal). Chitosan with 91.23\% deacytelation degree was purchased from Golden-Shell Biochemical Co., Ltd. (China). Calcium chloride $\left(\mathrm{CaCl}_{2}\right)$ was obtained from Panreac (Panreac Quimica SA, Barcelona, Spain) and vitamin $\mathrm{B}_{2}$ $(\mathrm{MW}=376.36 \mathrm{~g} / \mathrm{mol}$ ) was obtained from Sigma-Aldrich Chemical Co. Ltd. (St. Louis, MO, USA).

Phosphate buffer saline (PBS) was purchased Sigma-Aldrich Chemical Co. Ltd. (St. Louis, MO, USA), Potassium chloride was obtained from Merck (Darmstadt, Germany) and hydrochloric acid (36.5-38.0\% purity) was obtained from Sigma-Aldrich Chemical Co. Ltd. (St. Louis, MO, USA).

\subsection{Alginate/chitosan nanoparticles preparation}

The development of alginate/chitosan nanoparticles was based on the methodology described by Sarmento et al. [16] with some modifications. Briefly, sodium alginate was dissolved in distilled water and chitosan was dissolved in $1 \%$ of acetic acid being the $\mathrm{pH}$ values of alginate and chitosan solutions initially set to 4.9 and 4.6, respectively. The $\mathrm{pH}$ values of the solutions were adjusted with sodium hydroxide (1 M) and hydrochloric acid (1 M). Through a preliminary study, the optimal concentrations (selected based in the particle size and polydispersity index (PDI) using dynamic light scattering) of alginate and chitosan were determined, being, respectively, $0.63 \mathrm{mg} / \mathrm{mL}$ and $0.4 \mathrm{mg} / \mathrm{mL}$. Initially, $7.5 \mathrm{~mL}$ of $18 \mathrm{mM}$ calcium chloride solution was dropped at a flow rate of $0.125 \mathrm{~mL} / \mathrm{min}$ into a beaker containing $117.5 \mathrm{~mL}$ of alginate solution at a constant homogenization using an Ultra-Turrax ( $\mathrm{T} 25$, IkaWerke, Germany) at 20,000 rpm. Then, $25 \mathrm{~mL}$ of chitosan solution were added dropwise into the previous solution with a stirring of $600 \mathrm{rpm}$ and a flow rate of $0.278 \mathrm{~mL} / \mathrm{min}$. After the addition of chitosan solution, alginate/chitosan nanoparticles were maintained at constant stirring during $30 \mathrm{~min}$. For the water-soluble vitamin encapsulation, vitamin $\mathrm{B}_{2}$ was dissolved in the alginate solution before addition of calcium chloride $\left(\mathrm{CaCl}_{2}\right)$ solution. Concentrations of vitamin $B_{2}$ were tested based on their maximum solubility in water, then the optimal concentration of vitamin $B_{2}$ was determined based in particle size and PDI, being $0.065 \mathrm{mg} / \mathrm{mL}$. After preparation, the alginate/chitosan nanoparticles with or without vitamin $B_{2}$ were stored at $4{ }^{\circ} \mathrm{C}$ in solution.

\subsection{Nanoparticle size, polydispersity index and zeta potential}

Alginate/chitosan nanoparticles with and without vitamin $B_{2}$ were characterized in terms of size (by number distribution), the polydispersity index (PDI) and zeta potential. The particle size is the diameter of nanoparticles and PDI is the parameter that gave us the distribution of nanoparticles size. The PDI is dimensionless and indicate that the sample is or not monodisperse, in other words, has a very broad size distribution. The measurements of these parameters are based in a simple principle: illuminating of sample with a laser and analyzing the scattered light, where the detector position can be at either $173^{\circ}$ or $90^{\circ}$ depending on the model of Zetasizer Nano model, in our case were measured with a detection angle of $173^{\circ}$. The zeta potential $(\zeta)$ is other parameter that can be measurement by DLS and consist, essentially, in measurement of electrostatic/charge at the surface of the nanoparticle through a laser that passes the sample cell.

For this characterization a dynamic light scattering (DLS) apparatus (Zetasizer Nano ZS, Malvern Instruments, UK) equipped with a He-Ne laser at a wavelength of $633 \mathrm{~nm}$ was used. All measurements were performed at $25^{\circ} \mathrm{C}$. Each measurement of size and PDI was performed with a detection angle of $173^{\circ}$ and zeta potential measurements with an angle of $17^{\circ}$, being the zeta potential values calculated by Smoluchowski's model. For all size, PDI and zeta potential measurements at least three replicates were obtained.

NanoSight NS500 (NanoSight Ltd., UK) was also used to measure the size of nanoparticles and to analyze the nanoparticles concentration by size. For measurements the samples were diluted to a concentration suitable for the analysis (according to the equipment instructions) (NanoSight, 2010). The dilutions were made with distilled water. The diluted samples were injected into the sample chamber fitted with a $640 \mathrm{~nm}$ diode laser. The software Nanoparticle Tracking Analysis (NTA) 2.0 Build 127 was used for capturing and analyzing the data.

\subsection{Morphology}

The surface morphology of the nanoparticles with and without vitamin was evaluated by transmission electron microscopy (TEM) (ZEISS 902 A, Zeiss, Germany) operating at a voltage of $80 \mathrm{kV}$. Before loading of samples into the microscope (direct deposition), the samples were drop-cast onto a carbon coated copper grid and dried during $1 \mathrm{~min}$ at room temperature.

\subsection{Encapsulation efficiency and loading capacity}

Encapsulation efficiency (EE) and loading capacity (LC) were determined after separating the nanoparticles with encapsulated vitamin from the supernatant with free vitamin. The separation was performed using an Amicon ${ }^{\circledR}$ Ultra- 0.5 centrifugal filter device (Amicon ${ }^{\circledR}$ Ultra - $0.5 \mathrm{~mL} 3 \mathrm{~K}$ device, Millipore Corp., Ireland). Briefly, $0.5 \mathrm{~mL}$ of sample was added to the Amicon ${ }^{\circledR}$ and was centrifuged at $14,000 \times \mathrm{g}$ during $10 \mathrm{~min}$. After centrifugation a filtrate with free vitamin and a concentrate with nanoparticles with encapsulated vitamin were obtained. These were used for $\mathrm{EE}$ and LC determination. To calculate EE, the filtrate was assayed spectrophotometrically at $437 \mathrm{~nm}$, which corresponds to the 
maximum absorbance peak of vitamin $\mathrm{B}_{2}$ [23], and the amount of free vitamin was calculated using an appropriate calibration curve: $y=33.77 x-0.0049\left(R^{2}=0.999\right)$ being $y$ and $x$ the absorbance and amount of free vitamin $B_{2}$, respectively. LC was determined by the weight of dried nanoparticles $(0.5 \mathrm{~mL}$ of sample corresponds to approximately $0.7 \mathrm{mg}$ of nanoparticles). EE and LC were calculated as follows $[6,16]$ :

$E E \%=\frac{V i t_{\text {total }}-V_{\text {free }}}{\text { Vit }_{\text {total }}} \times 100$

$L C \%=\frac{V i_{\text {total }}-V_{\text {free }}}{N p s_{\text {total }}} \times 100$

where Vit $_{\text {total }}$ represents the total amount of vitamin; Vit free the amount of free vitamin in filtrate and $N p s_{\text {total }}$ the total weight of nanoparticles.

\subsection{Release profile}

Dialysis methodology was used in order to evaluate the release profile of encapsulated vitamins from nanoparticles [24-26]. The release experiments were conducted at 37 and $25^{\circ} \mathrm{C}$ and the release media utilized were PBS and Tris-HCL for $\mathrm{pH} 7$ and 2, respectively. The procedure of dialysis was as follows: $5 \mathrm{~mL}$ of nanoparticles (approximately $7 \mathrm{mg}$ of nanoparticles) with encapsulated vitamin were added into a dialysis membrane (molecular weight cut-off 3500 Da; Cell-Sep H1, Membrane Filtration products, USA) and the sealed membrane was then placed into $50 \mathrm{~mL}$ of medium (PBS or Tris- $\mathrm{HCl}$ ) at 37 or $25^{\circ} \mathrm{C}$, under magnetic stirring. At determined time intervals, $1 \mathrm{~mL}$ of samples were taken from the medium and replaced by fresh medium to maintain the original volume. All the experiments were performed in triplicate.

\subsubsection{Release kinetics}

The release profile of encapsulated vitamin in nanoparticles was evaluated using Berens and Hofenberg model [27] that considers contributions from Fickian diffusion and polymeric relaxations of the hydrophilic matrix:

$M_{t}=M_{t, F}+M_{t, F}$

being $M_{t}$ the total amount of sorption per unit weight of polymeric nanoparticles at time $t ; M_{t, F}$ the contributions of Fickian diffusion; and $M_{t, R}$ the contributions of polymeric relaxations.

In turn, for most cases, only one main polymer relaxation affects the transport and thus the equations can be simplified using $n=1$ [26]. This way the Fickian and relaxation contributions are expressed by the following equations:

$M_{t, F}=M_{F}\left[1-\frac{6}{\pi^{2}} \times \exp \left(K_{F} t\right)\right]$

$M_{t, R}=M_{R}\left[1-\exp \left(K_{R} t\right)\right]$

being,

$M_{t}=M_{F}\left[1-\frac{6}{\pi^{2}} \times \exp \left(K_{F} t\right)\right]+M_{R}\left[1-\exp \left(K_{R} t\right)\right]$

where,

$K_{F}=\frac{4 \pi^{2} D}{d^{2}}$

and $M_{F}$ the total mass of compound release at equilibrium by unrelaxed polymer; $M_{R}$ the total mass of compound release at equilibrium by relaxation process; $K_{R}$ the respective relaxation rate constant; $d$ nanoparticles diameter; and $D$ the diffusion coefficient.

The non-linear estimation module of Statistica ${ }^{\circledR} 7$ (Statsoft, Tulsa, OK, USA) was used in order to determine the suitable of equations in describing vitamin release data over time.

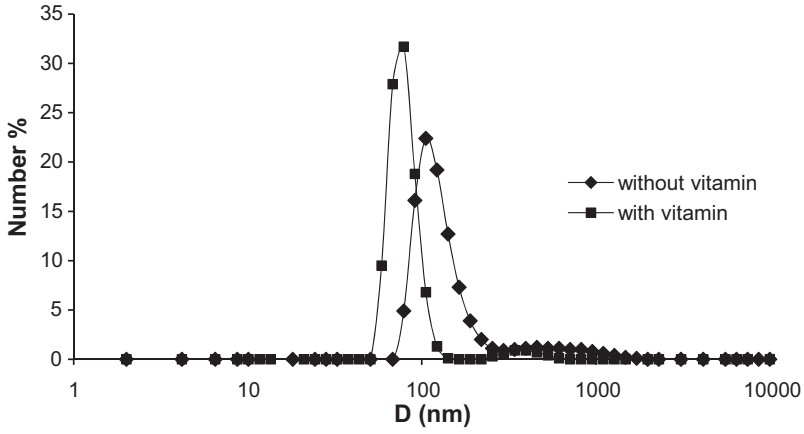

Fig. 1. Size distributions by number for alginate/chitosan nanoparticles without and with vitamin $B_{2}$.

\subsection{Stability}

Freshly prepared samples were used for stability measurements. In order to evaluate the stability of nanoparticles throughout time, nanoparticles with and without vitamin were stored at $4{ }^{\circ} \mathrm{C}$ in solution state being their size and PDI measured during five months.

\subsection{Statistical analyses}

Data analyses were performed using Microsoft Windows Excel 2003 and GraphPad Prism (Version 5.00 (Trial), edition 2007, GraphPad Software, Inc., La Jolla, CA, USA). Dispersion of data was reported in terms of the mean standard deviation (SD) from at least three values. Analysis of variance (ANOVA) followed by Tukey's multiple-comparison test $(\alpha=0.05)$ were employed to assess the statistical significance of differences between groups of data. Differences in experimental results were considered to be statistically significant at $95 \%$ confidence level $(p<0.05)$.

The quality of the regressions was evaluated on the basis of the determination coefficient, $R^{2}$, the squared root mean square error, RMSE (i.e., the square root of the sum of the squared residues (SSE) divided by the regression degrees of freedom) and residuals visual inspection for randomness and normality. $R^{2}$ was obtained directly from the software.

\section{Results and discussion}

\subsection{Diameter, polydispersity index (PDI) and zeta potential}

The diameter average of alginate/chitosan nanoparticles was $119.5 \pm 49.9 \mathrm{~nm}$ for samples without vitamin $B_{2}$ and $104.0 \pm 67.2 \mathrm{~nm}$ with vitamin $\mathrm{B}_{2}$, being the PDI values of $0.454 \pm 0.066$ and $0.319 \pm 0.068$, respectively (Fig. 1 ). The decrease of diameter values is not significantly different $(p>0.05)$ showing that encapsulation of vitamin does not influence the size of the nanoparticles, however the PDI values for alginate/chitosan nanoparticles without and with vitamin $\mathrm{B}_{2}$ are significantly different $(p<0.05)$ decreasing when the vitamin $B_{2}$ is loaded in alginate/chitosan nanoparticles. The lower values of PDI suggest that nanoparticles with vitamin $B_{2}$ are more homogeneous than samples without vitamin. This behavior can be explained by interactions between vitamin $B_{2}$ and biopolymers used. Vitamin $B_{2}$ when mixed with alginate solution at $\mathrm{pH} 4.7$, acquire a cationic form interacting strongly with negatively charged alginate [28]. We believe that this strong ionic interaction generated between vitamin $B_{2}$ and alginate is contributing to the decrease of both size and PDI in nanoparticles containing vitamin $\mathrm{B}_{2}$; also, the influence of hydrogen bonding and van der Waals forces that vitamin $B_{2}$ molecules can establish with alginate and chitosan must not be discarded [16]. The size of nanoparticles was also determined by NTA 


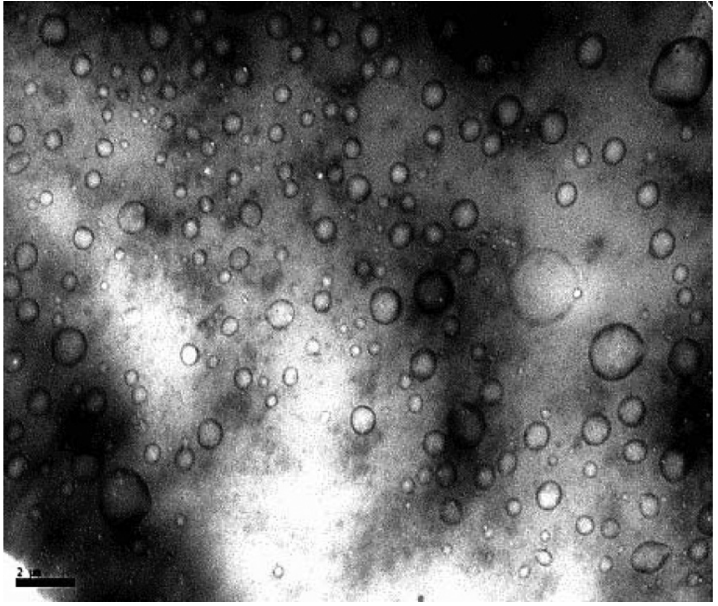

Fig. 2. Transmission electron microscopy (TEM) image of alginate/chitosan nanoparticles without vitamin $B_{2}($ scale bar $=2 \mu \mathrm{m}$, magnification $=7000 \times$ ).

in order to confirm the results obtained by DLS. The values of the mean diameter \pm standard deviation and the mode were respectively $168 \pm 97$ and $101 \mathrm{~nm}$, for nanoparticles without vitamin $\mathrm{B}_{2}$, and $210 \pm 109 \mathrm{~nm}$ and $107 \mathrm{~nm}$ for nanoparticles with vitamin $B_{2}$. The sizes obtained by NTA are consistent with the results obtained by DLS, confirming the nanoscale range of the developed nanoparticles.

When compared with others works (using similar methodologies and materials) the developed nanoparticles were shown to be smaller. Sarmento et al. [16] obtained sizes ranged between 700 and $4000 \mathrm{~nm}$, Zhang et al. [29] had a mean size around $750 \mathrm{~nm}$ for insulin-loaded alginate/chitosan nanoparticles and Goycoolea et al. [30] developed nanoparticles with diameter values ranging between 200 and $300 \mathrm{~nm}$. The same happens for other systems for encapsulation of vitamin $B_{2}$, e.g. El-Ghaffar et al. [31] obtained particles with significantly higher sizes $(1.40 \pm 0.05 \mathrm{~mm})$.

Zeta potential indicates if samples will have a good colloidal stability, being particles with zeta potentials above $+30 \mathrm{mV}$ or below $-30 \mathrm{mV}$ usually considered as being stable [32]. Besides that, zeta potential also provides information on the surface charge of nanoparticles. The values obtained were $-30.9 \pm 0.5$ and $-29.6 \pm 0.1 \mathrm{mV}$ for nanoparticles without and with vitamin $B_{2}$, respectively, showing that nanoparticles have a good colloidal stability. The zeta potential values are significantly different $(p<0.05)$ showing that vitamin-loading can influence the zeta potential of the nanoparticles; this behavior can be explained by the positive charge of vitamin $\mathrm{B}_{2}$ in solutions with low $\mathrm{pH}$ [28].

\subsection{Morphological observation}

Fig. 2 shows that alginate/chitosan nanoparticles have a spherical shape being the particle sizes in the same order of magnitude of values obtained by DLS and NTA. Moreover, Fig. 2 shows that particles' size distribution was not homogeneous, in agreement with the PDI values obtained.

\subsection{Encapsulation efficiency and loading capacity}

The ability of alginate/chitosan nanoparticles to encapsulate vitamin $B_{2}$ was evaluated through the determination of encapsulation efficiency (EE) and loading capacity (LC). The values of EE and LC obtained were $55.9 \pm 5.6 \%$ and $2.2 \pm 0.6 \%$, respectively. Other systems have shown higher values of EE for vitamin $B_{2}$ (80-100\%), however the size of those systems is significantly higher $(1.4 \pm 0.05 \mathrm{~mm})$ when compared with the size of the particles obtained in this work [31]. For other alginate/chitosan nanoparticles where different bioactive compounds (e.g. insulin) were encapsulated different results were observed: Zhang et al. [29] obtained EE between 58 and $80 \%$ and LC of 9.6\%, however the mean size obtained were greater than the mean size obtained in the present work; Sarmento et al. [16] obtained EE values between 70 and $90 \%$ and LC in the range $5-15 \%$, being the mean size of the particles between 700 and $4000 \mathrm{~nm}$; and Goycoolea et al. [30] obtained EE values similar to those obtained in this work ( $~ 50 \%)$, but also in this case the particles size are in the nanometric scale (200 and $300 \mathrm{~nm}$ ). This way, alginate/chitosan nanoparticles obtained in with this work presents good values of EE and LC.

\subsection{Release profile}

The release behavior of vitamin $B_{2}$ from alginate/chitosan nanoparticles was performed at 25 and $37^{\circ} \mathrm{C}$ and at $\mathrm{pH} 2$ and 7 , aiming at understanding how these conditions could influence particles' behavior at different external environment conditions (e.g. gastrointestinal conditions and acidic and alkaline food products).

A mathematical model (LSM, Eq. (6)) was applied to the experimental data (Fig. 3), and it is shown that the model-generated values are in good agreement with vitamin B2 release experimental data. This suggests that this model can be used to describe the physical mechanism involved in vitamin $B_{2}$ release from alginate/chitosan nanoparticles. From the parameters presented in Table 1 it can be seen that the transport mechanism involved in vitamin $B_{2}$ release does not involve Brownian motion alone, i.e. it does not strictly follow Fick's behavior, but is governed by both Fickian and relaxation phenomenon also called by Case II transport, with only one main relaxation of the nanoparticles. Also, regression analysis resulting of the LSM fitting showed that this model adequately describes the experimental data with relatively good regression quality $\left(R^{2}>0.90\right)$ and that almost all parameters were estimated with good precision.

Distinct release behaviors were observed for the tested conditions; explained by alginate and chitosan chemical structure the strength of electrostatic interactions between biopolymers at different $\mathrm{pH}$ values can lead to different release behaviors. The $\mathrm{p} K_{\mathrm{a}}$ value of alginate is around 3.5 [33], therefore at this $\mathrm{pH}$ the carboxylic groups are half protonated and consequently partially uncharged, which means that the electrostatic interactions between alginate and vitamin $\mathrm{B}_{2}$ or chitosan are weaker [23]. When submitted to $\mathrm{pH} 7$, a similar behavior can occur due to the $\mathrm{p} K_{\mathrm{a}}(7)$ of chitosan [33]. This leads to a change of nanoparticles' structure that promotes the release of vitamin $B_{2}$ due to polymer relaxation.

Regarding the Fick's component of transport, the total mass release from nanoparticles by Fick's mechanism $\left(M_{F}\right)$ is higher at $25^{\circ} \mathrm{C}$. This result can be explained by alginate and chitosan gelling properties, because the temperature is an external parameter that induces these properties. Alginate and chitosan can exhibit a solution or gel form when temperature is below or above $32^{\circ} \mathrm{C}$, respectively, being this temperature defined as the lower critical solution temperature (LCST) [34,35]. In addition to that, when a hydrophilic bioactive compound is loaded in alginate/chitosan particles and the temperature is below the LCTS, the total mass of bioactive compound release can increase. However, when temperature is above the LCTS, alginate and chitosan exhibit a gel form and preventing the release of the loaded component $[34,36]$. Considering the effect of $\mathrm{pH}$ on the mass release via Fick's mechanism, the experimental results show that for temperature $25^{\circ} \mathrm{C}$ the $M_{F}$ at $\mathrm{pH} 7$ and 2 are similar. Nonetheless, at $37^{\circ} \mathrm{C}$ the $M_{F}$ is lower at $\mathrm{pH} 2$ and this fact can be explained based on the effect of $\mathrm{pH}$ in vitamin $\mathrm{B}_{2}$ and the biopolymers used. As already mentioned, the vitamin $\mathrm{B}_{2}$ acquire a cationic form at $\mathrm{pH} 2$ interacting strongly with negatively charged alginate which slow down the diffusion of vitamin 

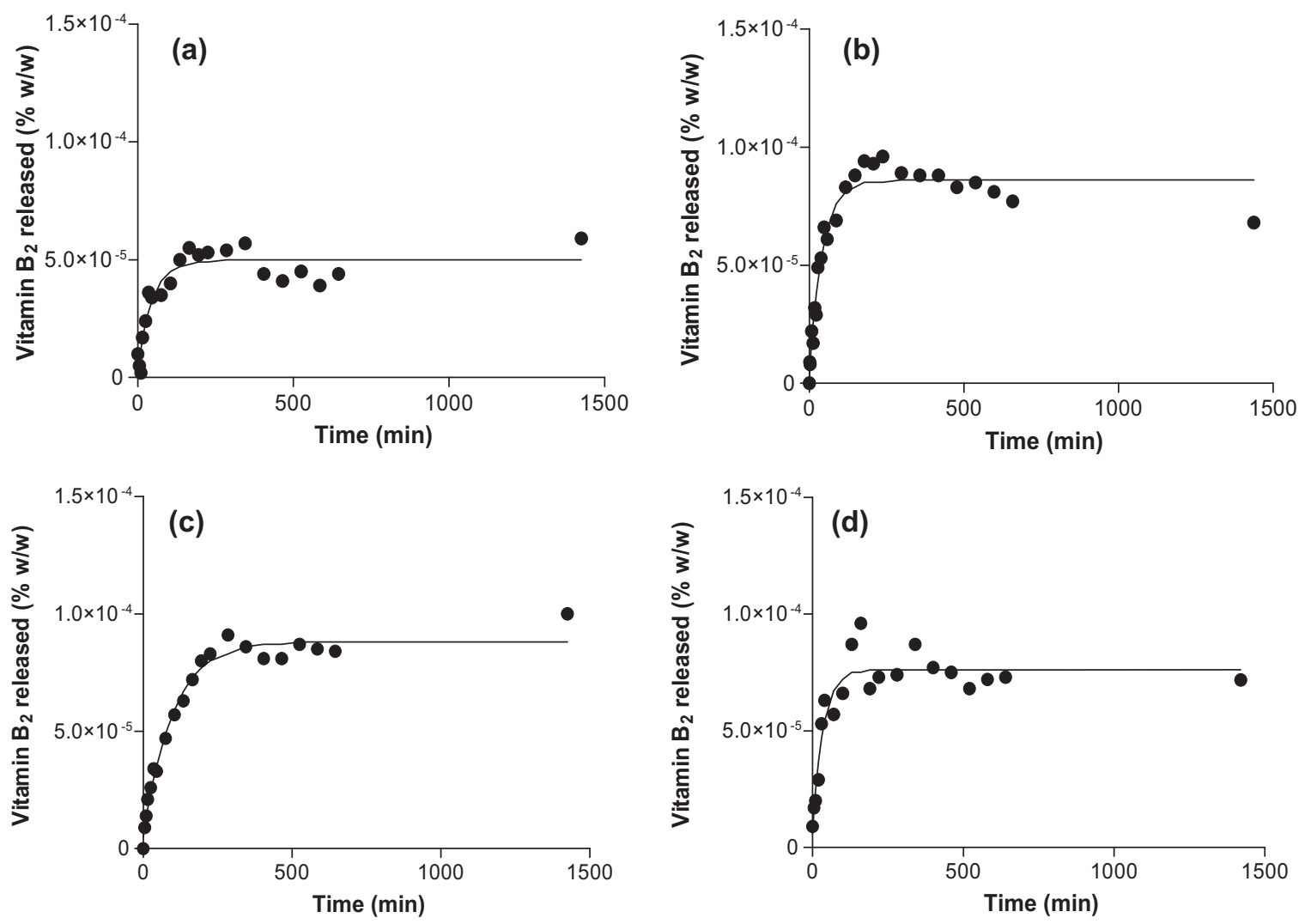

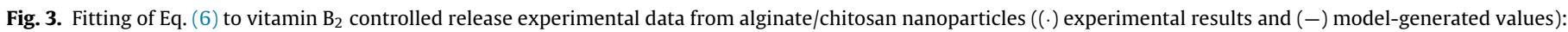
(a) at $37^{\circ} \mathrm{C}$ and $\mathrm{pH} 7$; (b) at $37^{\circ} \mathrm{C}$ and $\mathrm{pH} 2$; (c) at $25^{\circ} \mathrm{C}$ and $\mathrm{pH} 7$; (d) at $25^{\circ} \mathrm{C}$ and $\mathrm{pH} 2$.

$B_{2}$ into dissolution medium and consequently $M_{F}$ lower [28,31]. Other explanation for the lower $M_{F}$ at $\mathrm{pH} 2$ can be explained by the change of alginate structure at low pH's, which can lead to a shrink of the structure decreasing the release of vitamin $B_{2}$ encapsulated [37]. Nevertheless, the values of diffusion coefficient $(D)$ determined through Fickian rate of diffusion $\left(K_{F}\right.$, Eq. (7)) are very similar for all the tested conditions.

The total mass released from nanoparticles by relaxation $\left(M_{R}\right)$ presents a similar behavior when compared with total mass release by Fick's, being higher at $25^{\circ} \mathrm{C}$; happening the same for the relaxation rate constant $\left(K_{R}\right)$. Considering the effect of $\mathrm{pH}$ in relaxation parameters $\left(M_{R}\right.$ and $\left.K_{R}\right)$ it was observed that at $37^{\circ} \mathrm{C}, M_{R}$ is higher at $\mathrm{pH} 2$ and at $25^{\circ} \mathrm{C} M_{R}$ is higher at $\mathrm{pH} 7$ and for both temperatures the $K_{R}$ value is higher at $\mathrm{pH} 2$. When alginate/chitosan nanoparticles are placed in Tris- $\mathrm{HCl}$ buffer ( $\mathrm{pH} 2$ ), the negatively charged carboxylate groups of alginate begin to protonate to form uncharged $-\mathrm{COOH}$ groups. This reduces the degree of crosslinking due to the decrease of ionic and electrostatic interactions among the alginate and chitosan chains within the nanoparticles [23], this phenomenon may result in the loss of nanoparticle structure with subsequent faster release of vitamin $B_{2}$.

When compared the parameters $M_{F}$ and $M_{R}$, the experimental results have shown that for all tested conditions $M_{R}$ is higher than $M_{F}$. Being so, it can be concluded that Case II of transport (i.e. relaxation) is the mechanism prevailing in release profile of vitamin $B_{2}$ from alginate/chitosan nanoparticles.

\subsection{Stability}

Fig. 4 shows the average diameter values and PDI of alginate/chitosan nanoparticles during 5 months. Results showed that vitamin $B_{2}$-loaded nanoparticles are more stable in size than nanoparticles without vitamin $B_{2}$, being the PDI of alginate/chitosan nanoparticles with vitamin $B_{2}$ lower $(p<0.05)$ than that of nanoparticles without vitamin. This fact can be explained by interactions between vitamin $B_{2}$ and biopolymers used; vitamin $B_{2}$, besides the ionic interactions that can be established with

Table 1

Fitting the non-linear model (Eq. (6)) to experimental data of the release profile. Evaluation of the quality of the regression on the basis of RMSE and $R^{2}$.

\begin{tabular}{|c|c|c|c|c|c|c|}
\hline$T\left({ }^{\circ} \mathrm{C}\right)$ & $\mathrm{pH}$ & $R^{2}$ & $M_{F}$ & $D\left(\mathrm{~m}^{2} / \mathrm{s}\right)$ & $M_{R}$ & $K_{\mathrm{R}}\left(\min ^{-1}\right)$ \\
\hline \multirow[t]{2}{*}{37} & 7 & 0.871 & $\begin{array}{l}7.00 \mathrm{E}-06 \\
(78.13 \%)\end{array}$ & $\begin{array}{l}0.10 \mathrm{E}-12 \\
(0.0 \%)\end{array}$ & $\begin{array}{l}4.70 \mathrm{E}-05 \\
(0.0 \%)\end{array}$ & $\begin{array}{l}4.70 \mathrm{E}-05 \\
(99.86 \%)\end{array}$ \\
\hline & 2 & 0.950 & $\begin{array}{l}5.00 \mathrm{E}-06 \\
(80.77 \%)\end{array}$ & $\begin{array}{l}0.15 \mathrm{E}-12 \\
(25 \%)\end{array}$ & $\begin{array}{l}8.40 \mathrm{E}-05 \\
(0.0 \%)\end{array}$ & $\begin{array}{l}2.40 \mathrm{E}-02 \\
(21.26 \%)\end{array}$ \\
\hline \multirow[t]{2}{*}{25} & 7 & 0.980 & $\begin{array}{l}1.30 \mathrm{E}-05 \\
(0.0 \%)\end{array}$ & $\begin{array}{l}0.45 E-12 \\
(0.0 \%)\end{array}$ & $\begin{array}{l}8.31 \mathrm{E}-05 \\
(0.0 \%)\end{array}$ & $\begin{array}{l}1.02 \mathrm{E}-02 \\
(18.06 \%)\end{array}$ \\
\hline & 2 & 0.900 & $\begin{array}{l}1.59 \mathrm{E}-05 \\
(68.55 \%)\end{array}$ & $\begin{array}{l}0.10 \mathrm{E}-12 \\
(0.0 \%)\end{array}$ & $\begin{array}{l}6.99 E-05 \\
(0.0 \%)\end{array}$ & $\begin{array}{l}2.90 \mathrm{E}-02 \\
(32.91 \%)\end{array}$ \\
\hline
\end{tabular}

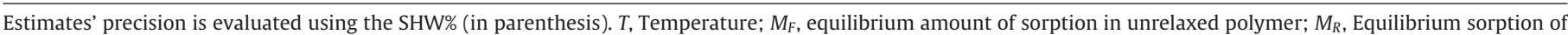
the relation process; $K_{R}$, relaxation rate constant; $D$, diffusion coefficient. 

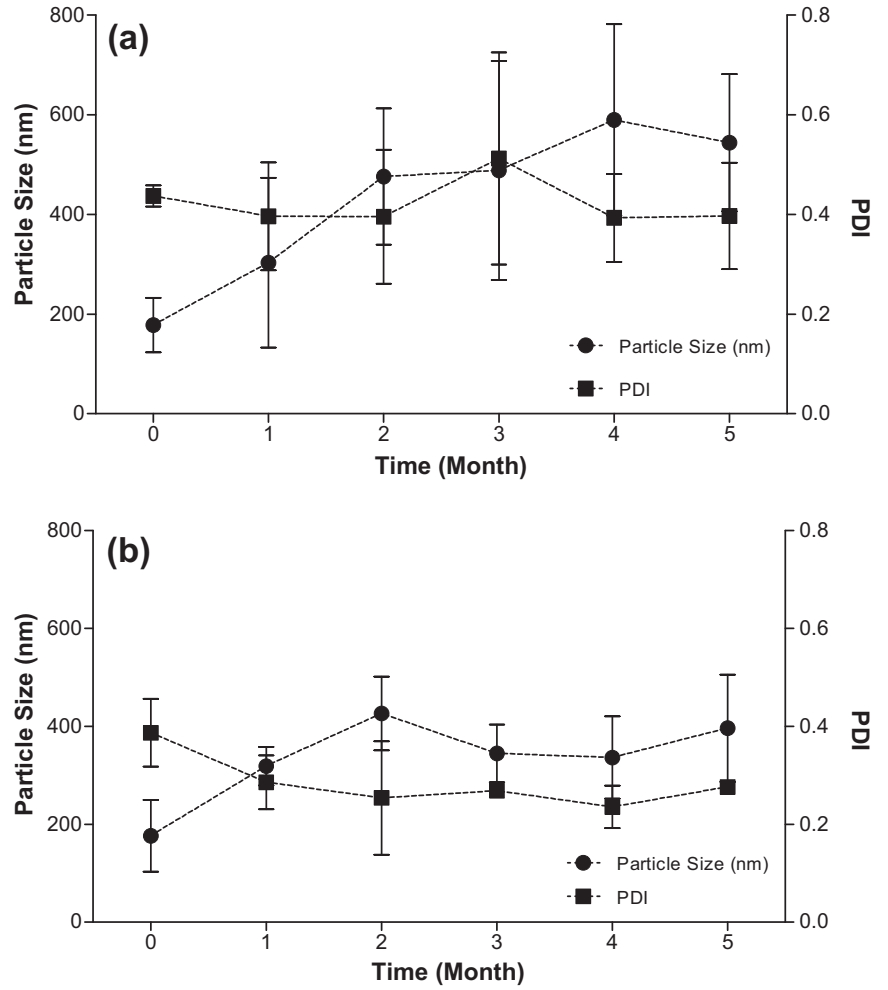

Fig. 4. Stability of alginate/chitosan nanoparticles: (a) without vitamin $B_{2}$; (b) with vitamin $B_{2}$.

alginate, can also interact via Van der Waals forces and hydrogen bonding with alginate and chitosan [16].

Nanoparticles were developed at $\mathrm{pH} 4.6$ near the alginate $\mathrm{p} K_{\mathrm{a}}$ value, being so the negatively charged carboxylate groups of alginate starts to protonate forming uncharged $-\mathrm{COOH}$ groups over time and the electrostatic interactions between alginate and chitosan became weaker [23]. As a result, the alginate can precipitate or aggregate [38] or the nanoparticle structure change [23] contributing to increase of the particle size.

It was also observed that the $\mathrm{pH}$ of medium increased from 4.6 to 5.4; this significant increase can be due to the change of alginate charge (explained above). This fact may also contribute to the instability of the nanoparticles and, consequently, the increase of nanoparticles' size [30,38].

Fig. 4 shows that alginate/chitosan nanoparticles without vita$\min B_{2}$ were more sensitive to alginate protonation and $\mathrm{pH}$ changes than alginate/chitosan nanoparticles with vitamin $B_{2}$, leading to higher values of size and PDI.

\section{Conclusion}

In this work has been demonstrated that encapsulation of vitamin $B_{2}$ in alginate/chitosan nanoparticles can be achieved using a pre-gelation ionotropic method, obtaining EE and LC values of $\sim 55 \%$ and $\sim 2 \%$, respectively. The release profiles showed that alginate/chitosan nanoparticles could be used in the retention of Vitamin $B_{2}$, being this system stable for at least five months. This work indicates that these nanostructures may be used in different food and/or beverage products for human nutrition, while contributing to increase vitamin stability in food matrices.

\section{Acknowledgements}

The authors Ana I. Bourbon and Miguel A. Cerqueira are recipients of a fellowship (SFRH/BD/73178/2010 and
SFRH/BPD/72753/2010, respectively), supported by Fundação para a Ciência e Tecnologia, POPH-QREN and FSE (FCT, Portugal). The authors thank the FCT Strategic Project PEst-OE/EQB/LA0023/2013 and the Project "BioInd - Biotechnology and Bioengineering for improved Industrial and Agro-Food processes", REF. NORTE-070124-FEDER-000028 Co-funded by the Programa Operacional Regional do Norte (ON.2-O Novo Norte), QREN, FEDER.

The support of EU Cost FA1001 is gratefully acknowledged. The authors would like to acknowledge to Rui Fernandes from IBMC University of Porto for his kind assistance in obtaining TEM pictures.

\section{References}

[1] G.F.M. Ball, Vitamins their Role in the Human Body, first ed., Blackwell Publishing Ltd., London, UK, 2008.

[2] G. Combs Jr., The Vitamins Fundamental Aspects in Nutrition and Health, fourth ed., Academic Press Ltd./Elsevier Science Ltd., USA, 2012.

[3] J.M. Berg, J.L. Tymoczko, L. Stryer, Biochemistry, third ed., W. H. Freeman and Company, New York, 2004

[4] W. Sebrell Jr., R. Harris, The Vitamins: Chemistry, Physiology, Pathology, first ed., Academic Press Inc., New York, 1954.

[5] D. de Britto, M.R de Moura, F.A. Aouada LH.C Mattoso, O.B.G. Assis, $\mathrm{N}, \mathrm{N}, \mathrm{N}-$ trimethyl chitosan nanoparticles as a vitamin carrier system, Food Hydrocolloids 27 (2012) 487-493.

[6] Y. Luo, Z. Teng, Q. Wang, J. Agric. Food Chem. 60 (2012) 836-843.

[7] Z. Fang, B. Bhandari, Trends Food Sci. Technol. 21 (2010) 510-523.

[8] C.E. Mora-Huertas, H. Fessi, A. Elaissari, Int. J. Pharm. 385 (2010) 113-142.

[9] P.N. Ezhilarasi, P. Karthik, N. Chhanwal, C. Anandharamakrishnan, Food Bioprocess Technol. 6 (2013) 628-647.

[10] S.L. Pal, U. Jana, P.K. Manna, G.P. Mohanta, R. Manavalan, J. Appl. Pharm. Sci. 1 (2011) 228-234.

[11] J.P. Rao, K.E. Geckeler, Prog. Polym. Sci. 36 (2011) 887-913.

[12] C. Chassenieux, D. Durand, P. Jyotishkumar, S. Thomas, Biopolymers: state of the art, new challenges, and opportunities, in: S. Thomas, D. Durand, C. Chassenieux, P. Jyotishkumar (Eds.), Handbook of Biopolymers-Based Materials: From Blends and Composites to Gels and Complex Networks, Wiley-VCH Verlag GmbH \& Co. KGaA, Germany, 2013, pp. 1-6

[13] C.M. Silva, J. Ribeiro, I. Vit, Int. J. Pharm. 311 (2006) 1-10.

[14] S. Sundar, J. Kundu, S.C. Kundu, Sci. Technol. Adv. Mater. 11 (2010) 0-14.

[15] S. Vrignaud, J. Benoit, P. Saulnier, Biomaterials 32 (2011) 8593-8604.

[16] B. Sarmento, A.J. Ribeiro, F. Veiga, D.C. Ferreira, R.J. Neufeld, J. Nanosci. Nanotechnol. 7 (2007) 1-9

[17] S.A. Agnihotri, N.N. Mallikarjuna, T.M. Aminabhavi, J. Control. Release 100 (2004) 5-28

[18] F. Shahidi, J.K.V. Arachchi, Y.J. Jeon, Trends Food Sci. Technol. 10 (1999) 37-51

[19] M.N.V.R. Kumar, React. Funct. Polym. 46 (2000) 1-27.

[20] V.K. Mourya, N.N. Inamdar, React. Funct. Polym. 68 (2008) 1013-1051.

[21] H. Peniche, N. Acosta, Chitosan: an attractive biocompatible polymer for microencapsulation, Macromol. Biosci. 3 (2003) 511-520.

[22] C. Weber, Biobased Food Packaging Materials for the Food Industry - Status and Perspectives, The Royal Veterinary and Agricultural University, Frederiksberg C, Denmark, 2000.

[23] S.K. Bajpai, R. Tankhiwale, React. Funct. Polym. 66 (2006) 1565-1574.

[24] S. Beirão da Costa, C. Duarte, A.I. Bourbon, A.C. Pinheiro, A.T. Serra, M. Moldão Martins, M.I. Nunes Januário, A.A. Vicente, I. Delgadillo, C. Duarte, M.L. Beirão da Costa, J. Food Eng. 110 (2012) 190-199.

[25] A.C. Pinheiro, A.I. Bourbon, A.A. Vicente, M.A.C. Quintas, J. Food Eng. 116 (2013) 633-638.

[26] A.C. Pinheiro, A.I. Bourbon, M.A.C. Quintas, M.A. Coimbra, A.A. Vicente, Innovat Food Sci. Emerg. Technol. 16 (2012) 227-232.

[27] A.R. Berens, H.B. Hopfenberg, Polymer 19 (May) (1978)

[28] E. Choe, R. Huang, D. Min, J. Food Sci. 70 (2005) 28-36.

[29] N. Zhang, J. Li, W. Jiang, C. Ren, J. Li, J. Xin, K. Li, Int. J. Pharm. 393 (2010) $212-218$.

[30] F.M. Goycoolea, G. Lollo, C. Remuñán-López, F. Quaglia, M.J. Alonso, Biomacromolecules 10 (2009) 1736-1743.

[31] M.A.A. El-Ghaffar, M.S. Hashem, M.K. El-Awady, A.M. Rabie, Carbohydr. Polym. 89 (2012) 667-675.

[32] M.S. Silva, D.S. Cocenza, R. Grillo, N.F.S. De Melo, P.S. Tonello, L.C. De Oliveira, D.L. Cassimiro, A.H. Rosa, L.F. Fraceto, J. Hazard. Mater. 190 (2011) 366-374.

[33] T.T. Khong, O.A. Aarstad, G. Skjåk-Bræk, K.I. Draget, K.M. Vårum, BioMacromolecules 14 (2013) 2765-2771.

[34] A.S. Carreira, F.A.M.M. Gonçalves, P.V. Mendonça, M.H. Gil, J.F.J. Coelho, Carbohydr. Polym. 80 (2010) 618-630.

[35] H.F. Liang, M.H. Hong, R.M. Ho, C.K. Chung, Y.H. Lin, C.H. Chen, H.W. Sung, Biomacromolecules 5 (2004) 1917-1925

[36] J.F. Mano, Adv. Eng. Mater. 10 (2008) 515-527.

[37] M. George, T.E. Abraham, J. Control. Release: Off. J. Control. Release Soc. 114 (2006) 1-14.

[38] B. Sarmento, D. Ferreira, F. Veiga, A. Ribeiro, Carbohydr. Polym. 66 (2006) $1-7$. 